\title{
Electroencephalographic evidence of organic alteration in a patient with SARS-CoV2 induced delirium
}

\author{
Jesus Pastor ${ }^{1,2 *}$, Lorena Vega-Zelaya ${ }^{1,2}$, Eduardo Delgado Parada ${ }^{3}$ and Jose L Ayuso Mateos ${ }^{2,3}$ \\ ${ }^{1}$ Department of Clinical Neurophysiology, Hospital La Princesa, Spain \\ ${ }^{2}$ Institute of Biomedical Research, Hospital La Princesa, Spain \\ ${ }^{3}$ Department of Psychiatry, Hospital La Princesa, Spain
}

\begin{abstract}
We have recently described, by means of quantified EEG (qEEG), a specific pattern in COVID-19 patients released from intensive care unit. In this work, we describe a patient showing an atypical delirium with insomnia, memory disturbances, temporo-spatial disorientation and significant language skills impairment, while maintaining a good level of attention and alertness. Three months later, the patient recovered its cognitive and behavioral premorbid state. $\mathrm{qEEG}$ was performed during delirium (EEGenc) and recovery (EEGcont) periods. Interestingly, during delirium, EEGenc was nearly normal by de visu inspection, but not for quantification. In fact, during illness the posterior dominant rhythm was slower $(8.5 \mathrm{~Hz})$ than recovery $(9.0 \mathrm{~Hz})$, with an excess of delta band at fronto-temporal regions and excessive synchronization in left hemisphere and right temporal lobe, moreover a high value of spectral entropy. Recovery was associated with a significant increase in theta and alpha bands of both hemispheres, together with beta band at frontal lobes. The relative normality of frontal lobes, together a most severe impairment of temporal ones, in the context of abnormal synchronization in the left hemisphere can explain the anomalous features of the delirium presented by the patient.
\end{abstract}

\section{Introduction}

Neurological complications in COVID-19 infected patients have been extensively reported. CNS affections include encephalitis, toxic encephalopathy, ageusia and anosmia, headache or acute cerebrovascular disease and delirium [1-5]. The mechanisms of CNS infection by CoV2 are still debated and it has been proposed a direct invasion through blood-brain barrier, a neuronal pathway, hypoxia damage, immune-response mediated injury or angiotensin-converter enzyme 2 , among others $[2,6,7]$.

COVID-19 patients suffering severe respiratory compromise are prone to develop neuropsychiatric symptoms [8]. Among these, one of the most frequent is the acute confussional syndrome or delirium (up to $28 \%)$ [5].

We have recently described, by means of quantified EEG (qEEG), a specific pattern in COVID-19 patients released from intensive care unit (ICU), showing brain alteration associated with neuropsychiatric symptoms [4].

In this work, we describe a patient with an atypical delirium and its evolution, highly correlated with alterations in qEEG, demonstrating an organic brain affection induced by COVID- 19 .

\section{Case presentation}

\section{Clinical case}

A 55-year-old male physician in active, with premorbid conditions of essential tremor without treatment and moderate alcoholic consumption, was directly admitted at intensive care unit (ICU) by acute severe respiratory syndrome (SARS-CoV-2), with a $86 \%$ minimum capillary oxygen saturation $\left(\mathrm{SaO}_{2}\right)$. The patient was intubated under sedo-analgesia (fentanyl, midazolam and dexmedetomidine) and treated with tocilizumab (3 doses), hydroxychloroquine (12 days), lopinavir/ritonavir (14 days) and levofloxacine (15 days). Fifteen days later was released to conventional hospitalization area. The patient developed a critically illness polyneuropathy, later resolved by rehabilitation. After five days of clinical stability, the patient developed significant cognitive and behavioral changes. Neurological examination did not uncover any further focal neurological deficits. He developed insomnia and an atypical delirium with memory disturbances, temporospatial disorientation and significant language skills impairment, while maintaining a good level of attention and alertness. The patient was treated with high doses of antipsychotics, initially aripiprazole (10 $\mathrm{mg} /$ day) and quetiapine ( $100 \mathrm{mg} /$ day), later replaced by risperidone $(6 \mathrm{mg} /$ day) when vascular damage was discarded. Imaging studies such as computerized tomography and magnetic resonance imaging were normal. EEG (EEGenc) was nearly physiologic in a first de visu inspection, although qEEG showed a mild encephalopathy (see below). HIV and syphilis serology resulted negative, vitamin complex B and thyroid function were normal. The patient was delivered from hospital one month after admission.

Three months later, the patient was re-evaluated and a new EEG was performed (EEGcont). He showed a complete cognitive recovery and had returned to his job as physician. Only a residual fatigability remained (after climbing stairs 1-2 floors).

${ }^{\star}$ Correspondence to: Jesus Pastor, Department of Clinical Neurophysiology, Hospital La Princesa, Spain, E-mail: jesus.pastor@salud.madrid.org

Key words: acute confusional syndrome, COVID-19, power spectrum, quantified EEG, synchronization

Received: December 03, 2020; Accepted: December 08, 2020; Published: December 10, 2020 


\section{Quantification of EEG}

EEGs' records were performed using a 32-channel digital system (EEG32U, NeuroWorks, XLTEK ${ }^{\circ}$, Oakville, Canada) with 19 electrodes placed according to a 10-20 international system. Recordings were performed at $512 \mathrm{~Hz}$ sampling rate, with a filter bandwidth of 0.5 to $70 \mathrm{~Hz}$ and notch-filter of $50 \mathrm{~Hz}$. Electrode impedances were usually below $15 \mathrm{k} \Omega$. Artifact-free periods (excluding electro-oculogram, muscular or movement in awake patient) were selected and exported in ASCII file to be quantified. The algorithm used has been previously published $[4,9]$. Briefly, areas under the power spectrum, obtained by fast Fourier transform, were computed for classical EEG bands defined as delta $(0.5-4.0 \mathrm{~Hz})$, theta $(4.0-8.0 \mathrm{~Hz})$, alpha $(8.0-13.0 \mathrm{~Hz})$ and beta $(13.0-30.0 \mathrm{~Hz})$. Shannon Spectral entropy (SSE) and Pearson's correlation coefficient $(\rho)$ for pair of electrodes were also calculated. One second windowing $10 \%$ superimposed were used to compute all of these measurements. Exported records were between 120 and 300 s, which allowed a minimum of 130 and a maximum of 330 windows to be computed. Numerical analysis of EEG recordings was performed with custom-made Matlab ${ }^{\circledR}$ R2019 software (MathWorks, Natic, MA, USA).

Statistical comparisons between groups were performed using the Student's t-test for data with normal distributions. Normality was evaluated using the Kolmogorov-Smirnov test. The Mann-Whitney Rank sum test. SigmaStat ${ }^{\circledR} 3.5$ software (SigmaStat, Point Richmond, CA, USA) and Matlab ${ }^{\star}$ were employed for statistical analysis.

The significance level was set at $\mathrm{p}=0.05$. Results are shown as the mean \pm SEM, except where otherwise indicated.

De visu EEGenc showed a near normal background, with presence of a low-frequent medium-voltage sharp waves at left temporal lobe. Diagnose of a mild encephalopathy was performed by qEEG (see below). The EEGcont performed three months later, showed a quite similar background without irritative activity at left temporal lobe. Nevertheless, the numerical analysis of EEGenc showed more interesting and no self-evident results. Spectral analysis revealed a generalized excess of delta bands, especially at anterior regions, with a posterior dominant rhythm at $8.5 \mathrm{~Hz}$ and a mean amplitude of 17.1 $\pm 1.7 \mu \mathrm{V}$. On the contrary, the numerical analysis EEGcont showed very different results. In fact, the posterior dominant rhythm was 9.0 $\mathrm{Hz}$, with the same amplitude $(21.1 \pm 1.3 \mu \mathrm{V}$, not significant, MannWhitney on ranks). In both cases, amplitude of EEG was normal and not reduced, as described in de visu analysis [10]. It's was quite surprising that not only the power of different bands were different between both recordings, with excess of delta band, mainly at frontotemporal regions, but it was observed that distribution of frequencies was different, with an increase in the peak frequency of alpha band (Figure $1 \mathrm{~A}, \mathrm{~B}$ ). Moreover, the pattern of EEG, defined as the percentage of different bands by lobe, resulted highly different. In fact, recovery is associated with a significant increase in theta and alpha bands of both hemispheres, together with beta band at frontal lobes (Figure $1 \mathrm{C}-\mathrm{E}$ ).

Nonetheless, not only cerebral rhythms were changed during recovery, but also the synchronization was modified (Table 1). Interestingly, during the encephalopathy the patient presented an excessive synchronization in left hemisphere and right temporal lobe (Table 1).

Another interesting fact described in encephalopatic COVID-19 patients is the relative increase in SSE. We have observed this fact for all the scalp regions in our patient and its decrease with recovery (Table 2).

\section{Discussion}

We have analyzed the scalp EEG performed in a patient during acute COVID-19 illness and three months later after clinical resolution and we have observed very relevant changes in $\mathrm{qEEG}$ among both periods that can be helpful to explain pathophysiology and can be used to diagnosis. Reports about EEG in COVID-19 patients are performed by de visu and different findings have been described but without specific features [10-12]. Nevertheless, we have described the EEG structure of encephalopathy in COVID-19 patients released from ICU after severe illness that clearly differs from other kinds of encephalopathy [4], showing specific features.

During encephalopathy, background EEG was scarcely pathological and the mild left temporal irritative activity observed cannot be responsible of the delirium. However, the brain activity was significantly impaired, as we observed in qEEG, e.g the cortical bands (alpha and beta [9]) were severely decreased, mainly at temporal and parieto-occipital lobes, together with an increase of theta activity, besides; delta activity was increased in temporal lobes, mainly in the right. Moreover, these modifications were associated with changes in synchronization and structure of band composition across the scalp, as indicated by SSE. The relative normality of frontal lobes, together a most severe impairment of temporal lobes, in the context of abnormal synchronization in the

Table 1. Comparison for Pearson's coefficient $(\rho)$ for EEGenc and EEGcont

\begin{tabular}{|c|c|c|c|c|c|}
\hline Location & & Total & Frontal & Parieto-occipital & Temporal \\
\hline \multirow{3}{*}{ Left hemisphere } & Encephalopathy & $0.514 \pm 0.003$ & $0.525 \pm 0.007$ & $0.495 \pm 0.007$ & $0.468 \pm 0.004$ \\
\hline & Control & $0.497 \pm 0.003$ & $0.498 \pm 0.003$ & $0.482 \pm 0.006$ & $0.610 \pm 0.008$ \\
\hline & Probability & 0.001 & 0.001 & n.s & 0.001 \\
\hline \multirow{3}{*}{ Right hemisphere } & Encephalopathy & $0.515 \pm 0.003$ & $0.526 \pm 0.007$ & $0.495 \pm 0.007$ & $0.468 \pm 0.004$ \\
\hline & Control & $0.508 \pm 0.005$ & $0.532 \pm 0.012$ & $0.510 \pm 0.009$ & $0.643 \pm 0.011$ \\
\hline & Probability & 0.05 & n.s & n.s & 0.001 \\
\hline
\end{tabular}

Table 2. Comparison of Shannon spectral entropy (SSE) for EEGenc and EEGcont

\begin{tabular}{|l|c|c|c|}
\hline Location & & Frontal & Parieto-occipital \\
\hline \multirow{3}{*}{ Left hemisphere } & Encephalopathy & $4.3 \pm 0.03$ & $4.60 \pm 0.03$ \\
\cline { 2 - 4 } & Control & $4.17 \pm 0.03$ & $4.91 \pm 0.04$ \\
\cline { 2 - 4 } & Probability & 0.001 & $4.28 \pm 0.04$ \\
\hline \multirow{3}{*}{ Right hemisphere } & Encephalopathy & $4.30 \pm 0.03$ & 0.04 \\
\cline { 2 - 4 } & Control & $4.08 \pm 0.03$ & $4.33 \pm 0.02$ \\
\cline { 2 - 4 } & Probability & 0.001 & $4.23 \pm 0.04$ \\
\hline
\end{tabular}


A
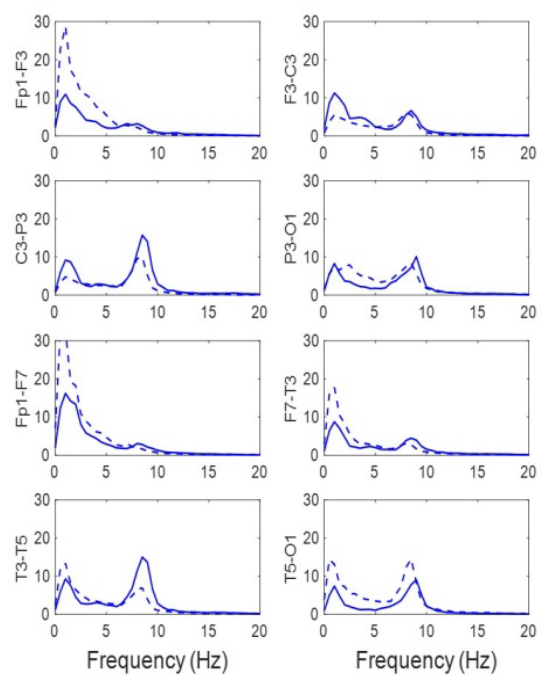

B
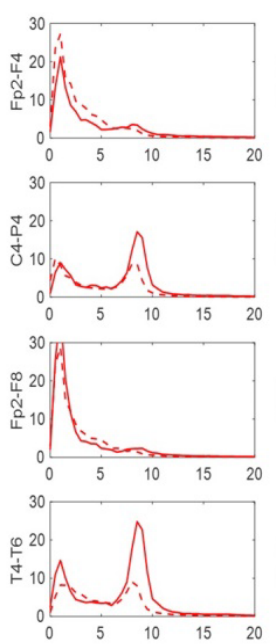

Frequency $(\mathrm{Hz})$
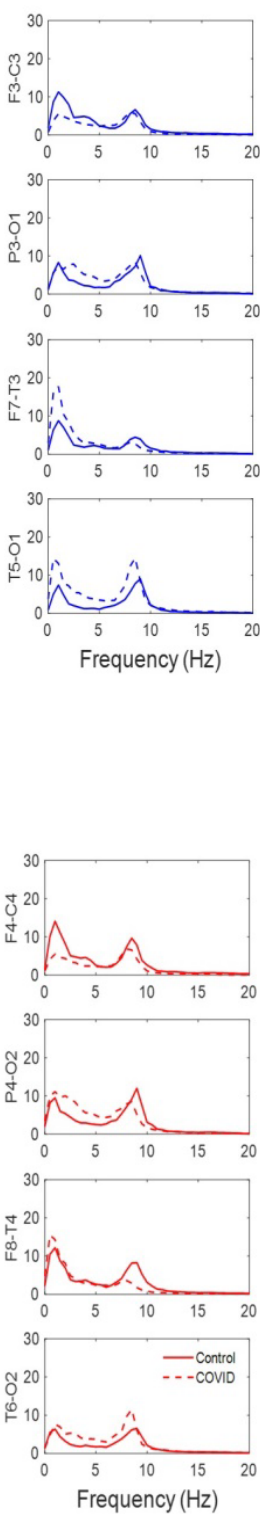
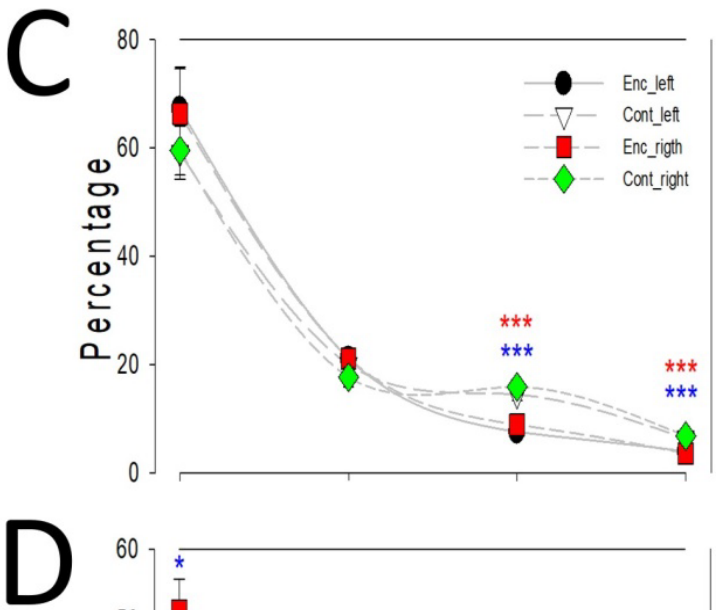

D
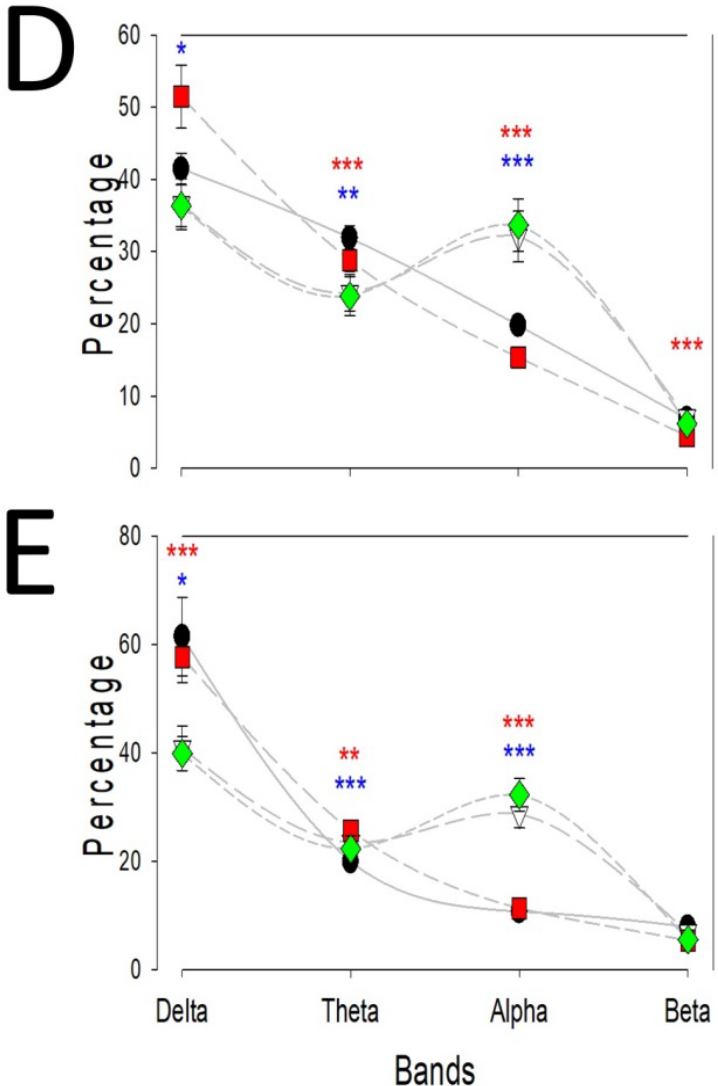

Figure 1. Numerical comparison between qEEG. (a) Mean spectra by channels of the left hemisphere and (b) right hemisphere. Dashed lines represent values from EEGenc and continuous lines EEGcont. Comparison of values for bands at (c) frontal, (d) parieto-occipital and (e) temporal lobes

Note: Filled circle=left encephalopathy; empty triangle=left control; red square=right encephalopathy and green diamond=right control. Blue asterisks=statistically significant difference for left hemisphere and red ones for right hemisphere. ${ }^{*} \mathrm{p}<0.05 ; * * \mathrm{p}<0.01 ; * * * \mathrm{p}<0.001$, Student t-test.

left hemisphere can explain the anomalous features of the delirium presented by the patient $[5,13,14]$.

We hypothesize that change affecting specific cerebral structures can be responsible of the different symptomatology described.

Considering that mild changes in synchronization or brain rhythmicity cannot be observed de visu, we encourage the use of objective measurements of bioelectrical variables to define more specific associations between electrical brain activity and symptoms in COVID-19 patients.

\section{Conclusion}

This is the first report of objective changes of bioelectrical brain activity during COVID-19 induced encephalopathy and after resolution. Reversible changes observed in EEG structure and connectivity can explain the anomalous delirium suffered by the patient.

\section{Conflicts of interest}

None. 


\section{Funding statement}

This work was partially financed (JP and LV-Z) by a grant from the Ministerio de Sanidad FIS [PI17/02193] and by Fonds Europeen de Developpement Economique et Regional (FEDER).

\section{References}

1. Sun T, Guan J (2020) Novel coronavirus and central nervous system. Eur J Neurol 27: e52. [Crossref]

2. Wu Y, Xu X, Chen Z, Duan J, Hashimoto K, et al. (2020) Nervous system involvement after infection with COVID-19 and other coronaviruses. Brain Behav Immun 87: 1822. [Crossref]

3. Scullen T, Keen J, Mathkour M, Dumont AS, Kahn L (2020) Coronavirus 2019 (COVID-19)-Associated encephalopathies and cerebrovascular disease: The New Orleans experience. World Neurosurg 141: e437-446. [Crossref]

4. Pastor J, Vega-Zelaya L, Martin Abad E (2020) Specific EEG encephalopathic pattern in SARS-CoV-2 patients. J Clin Med 9: 1545. [Crossref]

5. Zaragoza IA, Parada DE, Nuñez SM, Cruz AL, Corral MP, et al. (2020) Neuroinvasive hypothesis in a case of atypical delirium in a patient with pneumonia caused by COVID-19. Psiquiatría Biológica In press.

6. Chen C, Zhang XR, Ju ZY, He WF (2020) Advances in the research of cytokine storm mechanism induced by Corona Virus Disease 2019 and the corresponding immunotherapies. Zhonghua Shao Shang Za Zhi 36: 471-475.
7. Li Y, Fu L, Gonzales DM, Lavi E (2004) Coronavirus neurovirulence correlates with the ability of the virus to induce proinflammatory cytokine signals from astrocytes and microglia. J Virol 78: 3398-3406. [Crossref]

8. Mao L, Wang M, Chen S, He Q, Chang J, et al. (2020) Neurological manifestations of hospitalized patients with COVID-19 in Wuhan, China: A Retrospective Case Series Study. SSRN Electronic Journal In press.

9. Pastor J, Vega-Zelaya L, Martin Abad E (2020) Necessity of quantitative EEG for the daily practice. In: Electroencephalography. Ed: H Nakano InTech. ISBN 978-1-83968289-6. In press.

10. Pilato MS, Urban A, Alkawadri R, Barot NV, Castellano JF, et al. (2020) EEG Findings in Coronavirus Disease. J Clin Neurophysiol. [Crossref]

11. Petrescu A-M, Taussig D, Bouilleret V (2020) Electroencephalogram (EEG) in COVID-19: A systematic retrospective study. Neurophysiol Clin 50: 155-165. [Crossref]

12. Muccioli L, Pensato U, Cani I, Guerra L, Provini F, et al. (2020) COVID-19-related encephalopathy presenting with aphasia resolving following tocilizumab treatment. $J$ Neuroimmunol 349; 577400. [Crossref]

13. O'Hanlon S, Inouye SK (2020) Delirium: a missing piece in the COVID-19 pandemic puzzle. Age Ageing 2020: afaa094. [Crossref]

14. Sepulveda E, Franco J, Trzepacz P, Gaviria AM, Meagher DJ, et al. (2016) Delirium diagnosis defined by cluster analysis of symptoms versus diagnosis by DSM and ICD criteria: diagnostic accuracy study. BMC Psychiatry 2016: 167. [Crossref]

Copyright: (C2020 Pastor J. This is an open-access article distributed under the terms of the Creative Commons Attribution License, which permits unrestricted use, distribution, and reproduction in any medium, provided the original author and source are credited. 